TRANSACTIONS OF THE

AMERICAN MATHEMATICAL SOCIETY

Volume 365, Number 11, November 2013, Pages 5783-5804

S 0002-9947(2013)05782-0

Article electronically published on July 1, 2013

\title{
ADDITIVITY OF THE DP-RANK
}

\author{
ITAY KAPLAN, ALF ONSHUUS, AND ALEXANDER USVYATSOV
}

\begin{abstract}
The main result of this article is sub-additivity of the dp-rank. We also show that the study of theories of finite dp-rank cannot be reduced to the study of its dp-minimal types, and we discuss the possible relations between dp-rank and VC-density.
\end{abstract}

\section{INTRODUCTION}

This paper grew out of discussions that the authors had during a meeting at Oberwolfach in January 2010, following a talk of Deirdre Haskell, and conversations with Sergei Starchenko, on their recent joint work with Aschenbrenner, Dolich and Macpherson [2]. Haskell's talk made it apparent to us that the notion of VC-density (Vapnik-Chervonenkis density), investigated in [2], is closely related to "dependence rank" (dp-rank) introduced by the third author in [14. Discussions with Starchenko helped us realize that certain questions, such as additivity, which were (and still are, to our knowledge) open for VC-density, may be approached more easily in the context of dp-rank. This paper is the first step in the program of investigating basic properties of dp-rank and its connections with VC-density.

Whereas dp-rank is a relatively new notion, VC-density and related concepts have been studied for quite some time in the framework of machine learning, computational geometry, and other branches of theoretical computer science. Recent developments point to a connection between VC-density and dp-rank, strengthening the bridge between model theory and these subjects. We believe that investigating properties of dp-rank is important in discovering the nature of this connection. Furthermore, once this relation is better understood, theorems about dp-rank are likely to prove useful in the study of finite and infinite combinatorics related to VC-classes.

Dp-rank was originally defined in 14 as an attempt to capture how far a certain type (or a theory) is from having the independence property. It also helped us to isolate a minimality notion of dependence for types and theories (that is, having rank 1). We called this notion dp-minimality and investigated it in [7. Both dprank and dp-minimality were simplifications of Shelah's various ranks from [12] and

Received by the editors September 7, 2011 and, in revised form, December 21, 2011.

2010 Mathematics Subject Classification. Primary 03C45, 03C98; Secondary 05D99, 68R05.

Key words and phrases. Dp-rank, dp-minimality, VC-density.

The third author was supported by FCT grant SFRH/BPD/34893/2007 and FCT research project PTDC/MAT/101740/2008.

We thank the organizers of the meeting "Model theory: Around Valued Fields and Dependent Theories" at Oberwolfach in January 2010. Special thanks to Sergei Starchenko for valuable discussions during and after the meeting. We would also like to thank John Goodrick, Alfred Dolich, Pierre Simon and the anonymous referee for some very helpful remarks and comments on earlier drafts of this paper. 
from appropriate minimality notions. Our simplified notion of rank turned out to be very close to Shelah's $\kappa_{\text {ict }}$, but localized to a type.

A few characterizations of dp-minimality have since been given in the literature, and similar equivalences can also be applied to higher ranks. It seems clear at this point that part of the strength of this concept is the interaction between those equivalent definitions. We are mainly referring to the "standard" (syntactic) independent array definition as given in [14, 8] (see Definition 2.4), which is very useful when one wants to deal with formulas, and a very simple "semantic" variant (see Definition 2.1), which can be found, for the dp-minimal case, in Simon's paper (13]), but which (as far as we know) has never been stated for a type with rank greater than 1 . In this paper, we will mainly work with the semantic definition, which proves useful and convenient for our purposes. However, throughout the paper we prove quite a few different characterizations of dp-rank, especially in the finite rank case; we summarize them in Theorem 5.4.

As the word "rank" indicates, dp-rank is a certain measure of the "size" of a type. It would probably be more accurate to say that dp-rank measures "diversity" of realizations of the type - how much the realizations differ from each other, as can be seen by external parameters. We will elaborate on this below. Just as with most rank notions, one wonders whether it has basic properties such as subadditivity: the rank of a tuple should be bounded by the sum of the ranks of its elements. This is the main result of Section 4 . As a corollary, we obtain global bounds for alternation ranks of formulas in a theory of finite rank (so, in particular, in a dp-minimal theory).

Dp-rank is a notion which is related to weight in stable theories (and motivated by it) and to certain more recent notions of weight in dependent theories, e.g. 7], [8], and [6]. It is therefore natural to wonder whether dp-rank can play a role similar to weight in dependent theories. Although this is still a line of work which can be pursued (and probably provides natural lines of research), in this paper we give limitations to the analogy, showing that dp-rank fails to have certain properties that one would hope for in a notion of weight, in at least two significant ways.

One of our original intentions (influenced greatly by the weight analogy) was to prove results for dp-minimal types, and then try to extend them to higher ranks by induction, at least for the finite rank case. The analogy with weight in stable (and even simple) theories led us to ask whether every type of finite dp-rank can be "analyzed" to some extent by types of rank 1 . This turns out not to be the case and provides the first limitation to the weight comparison. Example 2.10 presents a theory with types of finite rank but no dp-minimal types. This is a very "tame" strongly dependent theory (hence of "finite weight"; in fact, of dp-rank 2), but there are no types of "weight" 1 (whatever definition of weight one chooses to use; see e.g. [7]).

Another direction where the analogy fails is the finite/infinite correspondence. Hyttinen's results in [4, that yield a decomposition of a type of finite weight in a stable theory into types of weight 1, also imply that no type has infinite but "rudimentarily finite" weight. In other words, if a type has weight $\aleph_{0}$, then there exists an infinite set witnessing this. In Subsection 2.2 (particularly, Theorem 2.13) we observe that this fails to be the case for dp-rank, even in stable theories. The example presented there also answers negatively an analogous question concerning Adler's notion of burden in 1 (since burden is essentially the same as dp-rank in 
dependent theories 1 ). Specifically, this is an example of an $\aleph_{0}$-stable theory (in particular, superstable) such that every type over $\emptyset$ has infinite dp-rank. Since in a superstable theory weight of any given complete type is finite, Theorem 2.13 exemplifies a very important difference between weight and dp-rank: weight only takes into account nonforking extensions of the type, so when passing to a forking extension, weight may grow. Dp-rank does not distinguish between different kinds of extensions, so it can only go down when the set of parameters is increased (as is the case with most rank/dimension notions).

In a sense, with the notion of dp-rank, we capture a very particular aspect of the size of the type, and our thesis is that it might be "better" to think of dp-rank in relation to finite combinatorial invariants of Vapnik-Chervonenkis (VC) classes, particularly, VC-density, instead of in terms of weight.

VC-density is one of the possible measures of growth rate of types over finite sets. Specifically, assuming that $\Delta(x, y)$ is a finite set of dependent formulas (which is equivalent to the growth rate of the number of finite $\Delta$-types being polynomial rather than exponential), VC-density bounds the degree of the corresponding polynomial. So VC-density 1 corresponds to linear growth of the number of $\Delta$-types over finite sets. We prove in Section 5 that one way of looking at dp-rank is the following: the dp-rank of a type $p$ corresponds to the degree of a polynomial that measures how many types a realization $c \models p$ can realize over finite indiscernible sequences. In other words, the dp-rank of $p$ is a measure of the number of realizations of $p$ that have different types over finite indiscernible sequences.

So, in particular, dp-minimality means that realizations of $p$ realize only an order of $n$ types over any given indiscernible sequence of length $n$. This is equivalent, as is shown in Section 2 to the following: subsets of indiscernible sequences that a realization of $p$ can definably pick are simply intervals. These intervals can be, of course, of length 1 (that is, singletons). The important observation here is that a dp-minimal element cannot pick (definably) a finite tuple of size larger than 1 from an arbitrary indiscernible sequence. Or, more precisely, its alternation rank cannot be larger than 2. In this respect the behavior of a dp-minimal element resembles an element in either an o-minimal theory or the theory of equality.

A number of distinctions between the notions of VC-density and dp-rank should be pointed out. The obvious ones are that we only count the number of types over indiscernible sequences (as opposed to arbitrary finite sets) and restrict ourselves to realizations of a given type (the second one is not important - one can look at the dp-rank of a partial type as well). Another difference is that, when calculating dp-rank, we do not restrict the set of formulas that one is allowed to use to a finite set. This is why, even in a strongly dependent theory, one can end up with types of dp-rank $\omega$ (one obtains more and more types over indiscernible sequences by changing the formulas), one more thing that Theorem 2.13 exemplifies.

However, dp-rank is a very natural model theoretic analogue of VC-density. Part of the strength of model theoretic techniques is the ability to approximate complex phenomena in better behaved structures. Indiscernible sequences have already proved very helpful for such approximations in various contexts. Seeking connections between VC-density and dp-rank is another implementation of this idea.

\footnotetext{
${ }^{1}$ If either is finite, then the two coincide; for infinite cardinals the dp-rank of a type may be the successor of the burden of the type.
} 
Since we work with the semantic definition of dp-rank, we need some technical results on indiscernible sequences which are quite interesting on their own. In Section 3 we prove a proposition (Proposition [3.3) which provides a "consistent" way to extend indiscernible sequences in an arbitrary theory. Specifically, in the proofs in Section 4 we are sometimes faced with the following situation: a sequence $I$ is indiscernible over various subsets of a set $B$, but not (necessarily) over $B$, and we would like a uniform way of extending $I$ to a longer sequence with the same properties. If the theory is assumed to be dependent (and $I$ is unbounded) one can just take the average type of $I$ over $B$. However, we are not assuming dependence, and it seems of independent interest to find a general technique allowing this (and more) in an arbitrary theory. Here Shelah's general notion of average type with respect to an ultrafilter comes in handy.

1.1. Structure of the paper. We begin Section 2 with definitions, characterizations, and basic properties. Then we proceed to a few examples, which point out what one can and cannot expect from dp-rank.

In Section 3 we develop a consistent way of extending mutually indiscernible sequences, which serves us in Section 4 .

In Section 4 we will prove the main result of the paper, the sub-additivity of the dp-rank. We start with the dp-minimal case, then proceed to types of finite rank, and finally the infinite case. There was no need, in fact, to give a separate proof for dp-minimal types: the general finite case can be easily modified to include rank 1 as well. However, the proof for dp-minimal types is much less involved, so we include it in order to exemplify the general principles that are used, and then proceed to the finite case by induction. The proof for infinite ranks is different, but easier - infinite combinatorics are more flexible and call for much less precise computations. We conclude the section with some corollaries such as global bounds on alternation ranks of formulas.

In Section 5 we discuss what is known about the relation between VC-density and dp-rank. Theorem 5.4 summarizes all the main equivalent ways of looking at dp-rank. We also pose a few questions and set up a framework for future work.

Throughout the paper, we will work in a monster model of an arbitrary first order theory $T$. In particular, although the main object of study is a "dependence rank", at no point do we assume that $T$ is dependent.

We will not distinguish between singletons and finite tuples in the notation. For example, when writing $\varphi(x)$ or " $a$ is in the sort of $x$ ", if not specified otherwise, $x$ and $a$ could be tuples.

\section{Dp-RAnK: Definitions AND BASiC PRoperties}

We begin with the definition of the main notion investigated in this paper.

Definition 2.1. Let $p(x)$ be a partial (consistent) type over a set $A$. We define the dp-rank of $p(x)$ as follows.

- The dp-rank of $p(x)$ is always greater than or equal to 0 . Let $\mu$ be a cardinal. We will say that $p(x)$ has dp-rank $\leq \mu$ (which we write as $\operatorname{rk}-\operatorname{dp}(p) \leq \mu)$ if given any realization $a$ of $p$ and any $1+\mu$ mutually $A$-indiscernible sequences, at least one of them is indiscernible over $A a$.

- We say that $p$ has dp-rank $\mu$ (or rk-dp $(p)=\mu$ ) if it has dp-rank $\leq \mu$, but it is not the case that it has dp-rank $\leq \lambda$ for any $\lambda<\mu$. 
- We call $p$ dp-minimal if it has dp-rank 1.

- We call $p$ dependent if a dp-rank of $p$ exists, that is, if it is an ordinal. In this case we write $\operatorname{rk}-\mathrm{dp}(p)<\infty$. Otherwise we write $\operatorname{rk}-\mathrm{dp}(p)=\infty$.

- We call $p$ strongly dependent if $\operatorname{rk}-\operatorname{dp}(p) \leq \omega$.

Remark 2.2. It may seem that dp-rank of $p$ depends on the set $A$; however, we will see in Remark 2.7 below that this is not the case. Hence all the notions in Definition 2.1 above are well defined.

The following is easy and standard.

Remark 2.3. The following hold for any (partial) type $p(x)$ and a set $A$.

(i) $p$ has dp-rank 0 if and only if it is algebraic.

(ii) $p$ is dependent if and only if the $\operatorname{rk}-\operatorname{dp}(p) \leq|T|^{+}$.

(iii) If $p$ is a type over a set $A$ and if $p^{\prime}$ is a type over a set $B \supseteq A$ that extends $p$, then $\operatorname{rk}-\mathrm{dp}\left(p^{\prime}\right) \leq \operatorname{rk}-\mathrm{dp}(p)$.

In particular, if $p$ is dp-minimal, then any extension of it is either dp-minimal or algebraic.

Definition 2.1 is a nice semantic characterization of the notion of dp-rank. We find it more convenient for the purposes of this paper than the syntactic definition in [8]. It is also much easier to grasp, in case one is unfamiliar with the concept. However, when working with formulas, it is useful to have a more syntactic notion, and we would like to prove that our "soft" characterization is equivalent to the original one. In case the reader is unwilling to deal with technical concepts, it is possible to skip the following definition and Proposition 2.6 in the first reading. These will rarely be used in the main body of the paper (Sections 3 and 4), but they are key for understanding important characterizations of dp-rank in the finite case and the connection to VC-density (Proposition 2.8 and Section [5), as well as the fact that dp-rank of a type does not depend on the set over which it is calculated.

The following definitions were motivated by the original definition of strong dependence by Shelah (see e.g. 12]) and appear in [14] and [7.

Definition 2.4. A randomness pattern of depth $\kappa$ for a (partial) type $p(x)$ over a set $A$ is an array $\left\langle b_{i}^{\alpha}: i<\omega\right\rangle_{\alpha<\kappa}$ and formulae $\varphi_{\alpha}\left(x, y_{\alpha}\right)$ for $\alpha<\kappa$ such that:

(i) the sequences $I^{\alpha}=\left\langle b_{i}^{\alpha}\right\rangle_{i<\omega}$ are mutually indiscernible over $A$; that is, $I^{\alpha}$ is indiscernible over $A I^{\neq \alpha}$,

(ii) length $\left(b_{i}^{\alpha}\right)=\operatorname{length}\left(y_{\alpha}\right)$,

(iii) for every $\eta \in{ }^{\kappa} \omega$, the set

$$
\Gamma_{\eta}=\left\{\varphi_{\alpha}\left(x, b_{\eta}^{\alpha}\right)\right\}_{\alpha<\kappa} \cup\left\{\neg \varphi_{\alpha}\left(x, b_{i}^{\alpha}\right)\right\}_{\alpha<\kappa, i<\omega, i \neq \eta(\alpha)}
$$

is consistent with $p$.

We will omit $A$ if it is clear from the context.

The following is standard.

Lemma 2.5. Let $p$ be a (partial) type over a set $A$. Then there is a randomness pattern of depth $\kappa$ over $A$ if and only if there exists an array $\left\langle b_{i}^{\alpha}: i<\omega\right\rangle_{\alpha<\kappa}$ and formulae $\varphi_{\alpha}\left(x, y_{\alpha}\right)$ for $\alpha<\kappa$ that satisfy clauses (ii) and (iii) of Definition 2.4 .

Proof. Without loss of generality $A=\emptyset$ (note that $A$ contains the domain of $p$ ). Given an array satisfying clauses (ii) and (iii) of Definition 2.4. we may assume by 
compactness that all the $\kappa$ sequences $\left\langle b_{i}^{\alpha}\right\rangle$ are as long as we wish, $\left\langle b_{i}^{\alpha}: i<\lambda\right\rangle$ for $\lambda$ arbitrarily large. Applying Ramsey's Theorem and compactness, one obtains new sequences for which clause (i) of the definition of a randomness pattern holds as well.

One consequence of the lemma is the following: let $p$ be a type, and let $A, B$ be sets, both containing the domain of $p$. Then there is a randomness pattern of depth $\kappa$ for $p$ over $A$ if and only if there exists such a pattern over $B$.

In 8 . we defined dp-rank of a type $p(x)$ as the supremum of all $\kappa$ such that there is a randomness pattern of depth $\kappa$ for $p(x)$. The following proposition shows that Definition 2.1 above is equivalent to the original ones.

The first appearance of any such equivalence appeared for the dp-minimal case in Lemma 1.4 of [13. We do not know that anyone has generalized this, even for finite dp-ranks (or randomness patterns of finite depth).

Proposition 2.6. The following are equivalent for a complete type $p(x)$ over $A$. Notice that $\kappa$ below may be a finite cardinal.

(i) There is a randomness pattern of depth $\kappa$ for $p(x)$ over $A$.

(ii) It is not the case that the dp-rank of $p(x)$ is less than or equal to $\kappa$.

(iii) There exist a set $\mathcal{I}:=\left\{I^{j}\right\}:=\left\{\left\langle a_{i}^{j}\right\rangle_{i \in I} \mid j \in \kappa\right\}$ of $\kappa$ infinite mutually indiscernible sequences over $A$ and a realization $c$ of $p(x)$ such that for all $j$ there are $i_{1}, i_{2}$ such that $\operatorname{tp}\left(a_{i_{1}}^{j} / A c\right) \neq \operatorname{tp}\left(a_{i_{2}}^{j} / A c\right)$.

Proof. The proof of the equivalence of (ii) and (iii) in [13. works exactly for the general case. Also, it is clear that (i) implies (iii).

Assume (iii), and we will prove (i). Let $\mathcal{I}_{j}:=\left\langle a_{i}^{j}\right\rangle$, and we will assume that all such $I^{j}$ are indexed by $\mathbb{Z}$, and let $\varphi^{j}$ be the formula such that $\varphi^{j}\left(c, a_{i_{1}}\right)$ and $\neg \varphi^{j}\left(c, a_{i_{2}}\right)$ hold.

Claim 2.6.1. If $\left\{i \mid \varphi^{j}\left(c, a_{i}^{j}\right)\right\}$ is both coinitial and cofinal, then there is a subsequence $I_{0}^{j}$ of $I^{j}$ such that (after re-enumerating the elements) $I_{0}^{j}:=\left\langle a_{i}^{0, j}\right\rangle_{i \in \mathbb{Z}}$ and $\neg \varphi^{j}\left(c, a_{i}^{0, j}\right)$ holds if and only if $i=0$.

Proof. The construction of $I_{0}^{j}$ is immediate from the definition.

Notice that if in every sequence $I^{j}$ we have that either $\left\{i \mid \varphi^{j}\left(c, a_{i}^{j}\right)\right\}$ or $\{i \mid$ $\left.\neg \varphi^{j}\left(c, a_{i}^{j}\right)\right\}$ is coinitial and cofinal, then we can replace $I^{j}$ by the subsequence $I_{0}^{j}$ and (replacing $\varphi^{j}(x, y)$ for $\neg \varphi^{j}(x, y)$ if necessary) we would have an instance of (i).

Now, if for some $j$ we have that both $\left\{i \mid \varphi^{j}\left(c, a_{i}^{j}\right)\right\}$ and $\left\{i \mid \neg \varphi^{j}\left(c, a_{i}^{j}\right)\right\}$ are not coinitial and not cofinal, then we have that, for example, $\left\{i \mid \varphi^{j}\left(c, a_{i}^{j}\right)\right\}$ is coinitial and $\left\{i \mid \neg \varphi^{j}\left(c, a_{i}^{j}\right)\right\}$ is cofinal. In this case, if we define $\varphi_{0}^{j}:=\varphi^{j}\left(x, y_{1}\right) \wedge \neg \varphi^{j}\left(x, y_{2}\right)$ and $I_{0}^{j}$ as a sequence $\left\langle a_{2 i}^{j}, a_{2 i+1}^{j}\right\rangle_{i \in \mathbb{Z}}$, we would preserve the mutual indiscernibility, we would have instances of both $\varphi_{0}^{j}\left(c, a_{2 i}^{j}, a_{2 i+1}^{j}\right)$ and $\neg \varphi_{0}^{j}\left(c, a_{2 i}^{j}, a_{2 i+1}^{j}\right)$, and $\{i \mid$ $\left.\neg \varphi^{j}\left(c, a_{2 i}^{j}, a_{2 i+1}^{j}\right)\right\}$ would be coinitial and cofinal. Applying the claim to all such sequences, we would have an instance witnessing (i).

Remark 2.7. It follows from Lemma 2.5 and the equivalence of (i) and (ii) in Proposition 2.6 that dp-rank of a type does not depend on the set $A$ over which it is computed. In other words, the notion $\operatorname{rk}-\operatorname{dp}(p)$ in Definition 2.1 is well defined. 
In the finite rank case, we can prove another characterization of dp-rank in terms of a natural generalization of the notion of alternation rank. Given a formula $\varphi(x, y)$, we define the $p$-alternation rank of $\varphi(x, y)$ over $A$ as follows: $\operatorname{alt}_{A}^{p(x)}(\varphi(x, y))$ $\geq k$ if there exists an $A$-indiscernible sequence $I$ and $c \models p$ such that the truth value of $\varphi(c, y)$ has $k$ alternations in $I$. The $p$-alternation rank of $\varphi(x, y)$ over $A$ is the maximal $k$ (if exists) such that alt ${ }_{A}^{p(x)}(\varphi) \geq k$. As usual, if $p \in \mathrm{S}(A)$, we may omit $A$.

Proposition 2.8. The following are equivalent for a partial type $p(x)$ over $A$ and $k<\omega$ :

(i) $\operatorname{rk}-\mathrm{dp}(p) \geq k$.

(ii) There exists a formula $\varphi(x, y)$ and an A-indiscernible sequence $I$ in the sort of $y$ such that for every subset $I^{\prime} \subseteq I$ of size $k$ there is a $c \models p$ such that $\varphi(c, y) \cap I=I^{\prime}$.

(iii) There exists a formula $\varphi(x, y)$ such that $\operatorname{alt}_{A}^{p(x)}(\varphi(x, y)) \geq 2 k$.

All the indiscernible sequences in the proposition are presumed to be infinite. Notice also that what (ii) is essentially saying is that every $k$-tuple in $I$ is $\varphi(c, y)$ definable for some $c \models p$.

Proof. (i) $\Longrightarrow$ (ii). We will use the syntactic definition here. If $\operatorname{rk}-\operatorname{dp}(p) \geq k$, then by Proposition 2.6 there is a randomness pattern of depth $k$ for $p(x)$. That is, there are $c \models p$, formulas $\varphi_{1}\left(x, y_{1}\right), \ldots, \varphi_{k}\left(x, y_{k}\right)$ and $A$-mutually indiscernible sequences $I_{i}=\left\langle a_{j}^{i}: j\langle\omega\rangle\right.$ in the sort of $y_{i}$ (for $i=1, \ldots, k$ ) such that for every $j_{1}, \ldots, j_{k}$ there is $c=c_{j_{1}, \ldots, j_{k}}=p$ such that $\varphi_{i}\left(c, a_{j}^{i}\right)$ if and only if $j=j_{i}$.

Let $\varphi\left(x, y_{1} \ldots y_{k}\right)=\bigvee_{i=1}^{k} \varphi_{i}\left(x, y_{i}\right)$, and let $I$ be an $A$-indiscernible sequence in the sort of $y_{1} \ldots y_{k}$ defined as follows: $I=\left\langle a_{j}: j<\omega\right\rangle$, where $a_{j}=a_{j}^{1} \ldots a_{j}^{k}$.

Choose arbitrary $k$ distinct indices $j_{1}, \ldots, j_{k}$. It is easy to see that $c=c_{j_{1}, \ldots, j_{k}}$ is a realization of $p$ such that $\varphi\left(c, a_{j}\right)$ if and only if $j \in\left\{j_{1}, \ldots, j_{k}\right\}$, as required in (ii).

(ii) $\Longrightarrow$ (iii) is trivial.

(iii) $\Longrightarrow(i)$. Let $I$ be an $A$-indiscernible sequence and $c \models p$ such that $\varphi(c, y)$ has $\geq 2 k$ alternations in $I$. We may assume that the number of alternation is finite and in fact equal to $2 k$. We prove by induction on $k$ the following claim:

Claim. If $I$ is an $A$-indiscernible sequence of order type $\mathbb{Q}$ and $\varphi(c, y)$ has $2 k$ alternations in $I$, then there is a randomness pattern $\left(I^{\alpha}, \varphi^{\alpha}\right)$ of $\operatorname{depth} k$ for $\operatorname{tp}(c / A)$ with $I^{\alpha}$ being segments of $I$, and $\varphi^{\alpha}=\varphi$ or $\varphi^{\alpha}=\neg \varphi$ for all $\alpha$.

The base case $k=0$ is trivial. So now given $I=\left\langle a_{q}: q \in \mathbb{Q}\right\rangle$ and $c$ as in the claim, since the order type of $I$ is $\mathbb{Q}$, there is an infinite initial segment on which $\varphi(c, y)$ is constant. Assume for example that $\varphi(c, y)$ holds, and let $r \in \mathbb{R}$ be the minimal cut such that for any $q>r$, the truth value of $\varphi(c, y)$ has changed signs twice in the interval $(-\infty, q)$. Let $q>r$ be such that $\varphi\left(c, a_{q}\right)$ holds, and there is no sign change between $r$ and $q$. Let $I^{0}=\left\langle a_{<r}\right\rangle \frown\left\langle a_{(r, q)}\right\rangle$ and $\varphi^{0}=\varphi$. Notice that by indiscernibility and compactness, given any $a_{i} \in I^{0}$ the type

$$
\neg \varphi\left(x, a_{i}\right) \wedge \bigwedge_{j \in I^{0} \backslash\left\{a_{i}\right\}} \varphi\left(x, a_{j}\right)
$$

is consistent. 
By the induction hypothesis, since the order type of $I_{>q}=\left\langle a_{>q}\right\rangle$ is still $\mathbb{Q}$ and since $I_{>q}$ has at least $2 k-2$ alternations of $\varphi(c, y)$, we can find a randomness pattern $\left\{I^{\alpha}\right\},\left\{\varphi^{\alpha}\right\}$ for $c$ over $A$, for $\alpha=1, \ldots, k-1$ and $I^{\alpha}$ segments of $I_{>q}$. Now clearly $\left\{I^{\alpha}\right\}_{\alpha=0}^{k}$ are mutually $A$-indiscernible (since they are all segments of the same $A$-indiscernible sequence), and it is easy to see that $\left\{I^{\alpha}\right\}_{\alpha \leq k},\left\{\varphi^{\alpha}\right\}_{\alpha \leq k}$ is a randomness pattern for $c$ over $A$ of depth $k$.

This finishes the proof of the claim and of the theorem.

Corollary 2.9. Assume that in a theory $T$ every type $p(x)$ over $\emptyset$ in the sort of $x$ has $d p$-rank $\leq k$. Then for every formula $\varphi(x, y)$, the alternation rank of $\varphi(x, y)$ is bounded by $2 k+1$.

Proof. If $\operatorname{alt}(\varphi(x, y)) \geq 2 k+2$, then there is an indiscernible sequence in the sort of $y$ and some $c$ in the sort of $x$ that witness this. By (iii) $\Rightarrow(\mathrm{i})$ in Proposition 2.8, $\operatorname{rk}-\operatorname{dp}(\operatorname{tp}(c / \emptyset)) \geq k+1$.

We conclude this section with a few examples that illustrate things which cannot be expected from dp-rank. We will leave some of the technical details of the examples to the reader.

2.1. Theory of dp-rank $\mathbf{2}$ with no dp-minimal types. As we mentioned above, one might be drawn to thinking that all the study of theories of finite dp-rank (theories where all dp-ranks are finite) can be reduced to dp-minimal types. This, however, is not the case.

Example 2.10. Consider the theory of an infinite set with two dense linear orders $<_{1}$ and $<_{2}$, and take the model completion of it. This is, the theory of a structure $M$ in $\mathcal{L}:=\left\{<_{1},<_{2}\right\}$ such that any finite formula consistent with $<_{1}$ and $<_{2}$ being dense linear orders is realized in $M$.

Every one type has dp-rank 2, and there are no dp-rank 1 types:

First of all, the model completion exists and by definition is complete and has elimination of quantifiers in the language $\mathcal{L}:=\left\{<_{1},<_{2}\right\}$, so $\operatorname{tp}(a / A)$ can be understood by formulas of the form $x<_{1} a, x<_{2} a$, and $x=a$ for suitable choices of $a \in A$.

It is now not hard to show that given any set $A$, any 1-variable type $p(x) \in \mathrm{S}(A)$ has dp-rank 2. Given any such set and type, it is enough to find mutually $A$ indiscernible sequences $\left\langle a_{i}\right\rangle$ and $\left\langle b_{j}\right\rangle$ such that for every $k, \ell$ we have:

- The set

$$
p(x) \cup\left\{x>_{1} a_{i}\right\}_{i \leq k} \cup\left\{x<_{1} a_{i}\right\}_{i>k} \cup\left\{x>_{2} b_{j}\right\}_{j \leq \ell} \cup\left\{x<_{2} b_{j}\right\}_{j>\ell}
$$

is consistent.

For this, it is enough to find, for every $m<\omega$, sequences $\left\langle a_{i}\right\rangle$ and $\left\langle b_{j}\right\rangle$ for $i, j<m$ such that for every $k, \ell<m$ we have:

- The set

$$
p(x) \cup\left\{x>_{1} a_{i}\right\}_{i \leq k} \cup\left\{x<_{1} a_{i}\right\}_{i>k} \cup\left\{x>_{2} b_{i}\right\}_{i \leq \ell} \cup\left\{x<_{2} b_{j}\right\}_{j>\ell}
$$

is consistent.

Such $a_{i}$ and $b_{j}$ can be found by the definition of a model companion.

This implies that every type in this theory has dp-rank at least 2, and in particular that there are no dp-minimal types. On the other hand, it is easy to see that no one type (over any set) has dp-rank larger than 2 . 
2.2. Type of dp-rank $\omega$ in a theory with types of finite weight. People have asked whether or not strong dependence was equivalent to every type having finite dp-rank, in the same way that a stable theory is strongly dependent if and only if every type has finite weight (some people asked this for Adler's notion of burden [1, which is essentially the same question). Specifically, the question is whether or not it is possible to have randomness patterns of arbitrarily large finite depths but no randomness pattern of infinite depth for a given complete type $p(x)$ (once one forgets the type and just asks whether there is a strongly dependent theory with arbitrarily deep randomness patterns, the question becomes much easier).

The following provides an example showing that the above is possible even in stable theories.

Let

$$
S:=\{(m, n) \in \mathbb{N} \times \mathbb{N} \mid m<n\}
$$

and let $<_{S}$ (which we sometimes denote simply by $<$ for simplicity) be the partial order on $S$ defined by $\left(m_{1}, n_{1}\right)<_{S}\left(m_{2}, n_{2}\right)$ if and only if $n_{1}<n_{2}$.

If $s=(m, n)$, we say that $s$ is of level $n$, and write $\operatorname{lev}(s)=n$. Note that $s \leq t$ if and only if $s$ is of a smaller level than $t$ (or $s=t$ ). So $s \neq t$ is incomparable if and only if it is of the same level. Hence there are finitely many $t$ 's which are incomparable to a given $s$; in fact, the number is exactly the $\operatorname{lev}(s)$.

So we have:

Observation 2.11. The following hold for $\left(S, \leq_{S}\right)$.

(i) For any $s \in S$ there are finitely many $s^{\prime} \in S$ which are not greater than $s$.

(ii) For any $n \in \mathbb{N}$ there exists $s_{1}, \ldots, s_{n} \in S$ such that $s_{i}$ is incomparable to $s_{j}$ for all $1 \leq i \neq j \leq n$.

Now consider the theory $T_{\forall}$ in the language $\mathcal{L}:=\left\{E_{s}\right\}_{s \in S}$ which states that all $E_{s}$ are equivalence relations and for any $s \leq_{S} t$ we have

$$
\forall x, y, x E_{s} y \Rightarrow x E_{t} y \text {. }
$$

Let $T$ be the model completion of $T_{\forall}$, so in particular it is a complete theory with elimination of quantifiers.

$T$ is axiomatized by the following axioms. In order to make things more uniform, let us refer to equality as the unique $s$ of level -1 .

- Every class of every $E_{s}$ for $s \in S$ is infinite.

- Every $E_{s}$ has infinitely many classes.

- Whenever $-1 \leq n<m, \operatorname{lev}(s)=n, \operatorname{lev}\left(t_{1}\right)=\operatorname{lev}\left(t_{2}\right)=\ldots=\operatorname{lev}\left(t_{k}\right)=m$, and $A_{i}$ is an equivalence class of $E_{t_{i}}($ for $i=1, \ldots, k)$, then $\bigcap_{i=1}^{k} A_{i}$ contains infinitely many classes of $E_{s}$.

Even without proving the existence of the model completion, one can show directly, given the above axioms and using a standard back and forth argument, that $T$ is a complete theory with elimination of quantifiers.

Claim 2.12. $T$ is $\aleph_{0}$-stable.

Proof. For every (nongeneric) type $p$ over a countable model $M$, let lev $(p)$ be the least $n$ such that there exists $a \in M$ and $s$ of level $n$, such that the formula $x E_{s} a$ is in $p$. Since this determines which $E_{t}$ classes $p$ "chooses" for $s<t$, there are only finitely many equivalence relations left to "settle", specifically, all those $E_{t}$ for which $s \nless t$ (see Observation 2.11 above). It follows (by quantifier elimination) that 
there are countably many types of each given level $n$. Since there are countably many levels, we are done.

We can now show that $T$ is an example of a theory with nonfinite dp-rank and no randomness pattern of depth $\omega$ for the unique type $p(x)$ over the empty set.

Theorem 2.13. For any element $c$ and any natural number $n$, one can find $\mathcal{I}$ such that $\mathcal{I}$ is a set of $n$ mutually indiscernible sequences (over $\emptyset$ ), none of which is indiscernible over c. However, no such example can be found with $\mathcal{I}$ infinite.

Proof. Let $n$ be a natural number, and let $s_{1}, \ldots, s_{n}$ be incomparable elements of $S$ of level $n$ (see Observation 2.11). By the axioms of $T$ we can find $I_{1}, \ldots, I_{n}$ mutually indiscernible sequences of singletons with the following properties:

- Elements of $I_{i}$ are $E_{s_{j}}$-equivalent if and only if $j \neq i$,

- $c$ is $E_{s_{i}}$-equivalent to the first element of $I_{i}$ for all $i$.

Clearly, none of the $I_{i}$ 's is indiscernible over $c$, as required.

On the other hand, by Claim 2.12, $T$ is superstable and hence strongly dependent (this is very easy to see directly, but see [12]). So there is no infinite randomness pattern for any type in any model of $T$. (Alternatively, using quantifier elimination, one can easily give a direct proof that there is no infinite randomness pattern.)

Remark 2.14. In a similar fashion, one constructs an example of a theory which is not superstable, but still strongly dependent, with a type of dp-rank $\omega$. This is done by switching the "nesting order" of the equivalence relation in the example above; that is, we demand in the universal theory that for $s \leq_{S} t$ we have

$$
\forall x, y, x E_{t} y \Rightarrow x E_{s} y .
$$

Here one has to give a direct argument as to why there is no infinite randomness pattern. However, this is quite straightforward, using quantifier elimination, and we leave it to the reader.

Recall that in a strongly stable theory (strongly dependent and stable) every type has finite weight $([1,14])$. However, in both examples discussed above, the unique (nonalgebraic) type over $\emptyset$ has dp-rank $\omega$. So we have two examples of theories where every type has finite weight but there are no nonalgebraic types of finite dp-rank over $\emptyset$. In fact, the generic type over any set will also have dp-rank $\omega$ by a similar argument. In the example discussed in Remark 2.14 the situation is even more extreme, because almost all types have infinite dp-rank (besides the algebraic and the strongly minimal ones).

\section{EXtending INDISCERNIBLE SEQUENCES}

Since the main definition of the paper involves mutually indiscernible sequences, it would be nice to have certain tools for handling such "independent arrays". Specifically, we would like to have a "consistent" way of extending indiscernible sequences. Our technique will make use of Shelah's notion of average types with respect to ultrafilters, which provides a way of constructing co-heir extensions. In spite of its usefulness, this notion does not yet seem to be widespread in the model theoretic community.

First, we recall the following easy observation. 
Fact 3.1. Given a set $A$ and an infinite indiscernible sequence $\mathcal{I}=\left\langle a_{i}: i \in I\right\rangle$ over $A$, there exists a unique complete type $p$ over $A I$ such that if $a \models p$, then $I^{\frown}\langle a\rangle$ is indiscernible over $A$.

This fact provides a natural and standard way to extend indiscernible sequences. However, it will not always be good enough for us, since, as mentioned in the introduction, we will need to extend sequences preserving indiscernibility over different subsets.

This is why the definition of average types from Shelah becomes useful:

Definition 3.2. Let $I=\left\langle a_{i}\right\rangle$ be an indiscernible sequence and $\mathcal{U}$ an ultrafilter on the index set of $I$. Given any set $B$ we will define $\operatorname{Avg}_{\mathcal{U}}(I, B)$, the average type of $I$ over $B$ given by $\mathcal{U}$, as the unique complete type $p(x)$ such that for every formula $\varphi(x, y)$ and $b \in B$ we have

$$
\varphi(x, b) \in p(x) \Leftrightarrow\left\{i \mid \varphi\left(x, a_{i}\right)\right\} \in \mathcal{U} .
$$

This definition will allow us to prove the following. The proof requires some knowledge of ultrafilters.

Proposition 3.3. Let $I$ be an indiscernible sequence indexed by an order with no last element, and let $B$ be any set. Then, for any indexing set $\lambda$, there is an indiscernible sequence $I^{*}$ indexed by $\lambda$ such that $I^{\frown} I^{*}$ is indiscernible over $A$ whenever $A \subseteq B$ is a set such that I was already indiscernible over $A$.

Proof. We will prove the result for any finite $I^{*}$. The general case follows by compactness.

If we take $\mathcal{U}$ to be an ultrafilter over $I$ such that every set in $\mathcal{U}$ is unbounded in $I$, it follows from the definition that for any $A$ such that $I$ is indiscernible over $A$ and any $a_{0} \models \operatorname{Avg}_{\mathcal{U}}(I, A I)$, we have $I^{\frown}\langle a\rangle$ is indiscernible over $A$. Since the definitions imply that $A v g_{\mathcal{U}}(I, B I)$ extends $A v g_{\mathcal{U}}(I, A I)$ for any such $A \subset B$, any realization $a_{0}$ of the type $p(x):=\operatorname{Avg}_{\mathcal{U}}(I, B I)$ will be such that $I \frown\left\langle a_{0}\right\rangle$ is indiscernible over $A$. Inductively, it follows from the properties of average types that if we let

$$
a_{n+1} \models \operatorname{Avg}_{\mathcal{U}}\left(I, B I^{\frown}\left\langle a_{n}, a_{n-1}, \ldots, a_{0}\right\rangle\right),
$$

then $I^{\frown}\left\langle a_{n+1}, a_{n}, a_{n-1}, \ldots, a_{0}\right\rangle$ is an indiscernible sequence over $A$. This construction will therefore give us an indiscernible sequence $I^{\prime}$, indexed by $n$, such that $I^{\frown} I^{*}$ is indiscernible over any $A$ over which $I$ was indiscernible (for an arbitrarily large $n$ ). By compactness, given any indexing set $\lambda$, we can find an indiscernible sequence $I^{*}$ indexed by $\lambda$ such that $I \frown I^{*}$ satisfies the conclusion of the proposition.

Proposition 3.3 is the only instance where we will use average types. If unwilling to think about ultrafilters, the reader can just assume the existence of a way to extend indiscernible sequences given by Proposition 3.3

Corollary 3.4. Let $I$ and $J$ be infinite mutually indiscernible sequences over $A$ and let $B \supset A$. Let $I^{*}$ be as in Proposition 3.3 .

Then $I^{\frown} I^{*}$ and $J$ are mutually indiscernible over $A$.

Proof. It follows from Proposition 3.3 that $I^{\frown} I^{*}$ is indiscernible over $A J$. Now, if $J$ was not indiscernible over $I^{\frown} I^{*}$ there would be a finite tuple $\bar{a} \frown \bar{b}$ with $\bar{a} \in I$ and $\bar{b} \in I^{*}$ such that $J$ is not indiscernible over $A \bar{a} \bar{b}$. Since $I^{\frown} I^{*}$ was indiscernible over 
$A J$, and since $I$ is infinite, we know that there are some $\bar{a}^{\prime}, \bar{b}^{\prime} \in I$ such that

$$
\operatorname{tp}\left(\bar{a}^{\prime} \bar{b}^{\prime} / A J\right)=\operatorname{tp}(\bar{a} \bar{b} / A J) .
$$

But this would imply that $J$ is not indiscernible over $A I$, contradicting our hypothesis.

\section{Additivity of the DP-RANK}

In this section we will prove the (sub-)additivity of the dp-rank (Theorem 4.8), which is the main result of this paper.

4.1. Warm-up case: dp-minimal. The first technical lemma essentially deals with sub-additivity for dp-minimal types. It will also form the induction base for the general case. Although we could modify the proof of the general statement slightly so that it deals with rank 1 as well, we decided to include the simple base case explicitly, since it exemplifies the general technique that we are using.

Lemma 4.1. Let $a$ be any tuple such that $\operatorname{tp}(a / A)$ is $d p$-minimal, let $B \supset A$, and let $\mathcal{I}$ be a set of mutually $B$-indiscernible sequences. Then for any $n$, given any $n+1$ mutually $B$-indiscernible sequences in $\mathcal{I}$, at least $n$ of them are mutually indiscernible over $B a$.

Proof. We will do an induction on $n$. Since any extension of a dp-minimal type is dp-minimal (or algebraic), if $n=1$ there is nothing to prove.

Assume now that $\mathcal{I}:=\left\{I_{1}, \ldots, I_{n+1}\right\}$ is a set of mutually $B$-indiscernible sequences for $B \supset A$. By definition $\left\{I_{1}, \ldots, I_{n}\right\}$ is mutually indiscernible over $B I_{n+1}$ so we can, by the induction hypothesis, find $n-1$ of the $I_{j}$ 's which are mutually indiscernible over $B I_{n+1} a$. We may assume without loss of generality that $\left\{I_{1}, \ldots, I_{n-1}\right\}$ is mutually indiscernible over $B I_{n+1} a$. If $I_{n+1}$ was indiscernible over $\{a\} \cup B \cup \cup\left\{I_{1}, \ldots, I_{n-1}\right\}$ the sequence $\left\{I_{1}, \ldots, I_{n-1}, I_{n+1}\right\}$ would satisfy the conditions of the claim, so we may assume that this is not the case. Since nonindiscernibility can be witnessed by a finite sequence, we will assume for the rest of the proof that $I_{n+1}$ is not indiscernible over $B a \bar{b}$ for some $\bar{b} \in \bigcup\left\{I_{1}, \ldots, I_{n-1}\right\}$, and that $\left\{I_{1}, \ldots, I_{n-1}\right\}$ is mutually indiscernible over $I_{n+1} B a$.

Claim 4.1.1. It is enough to prove Lemma 4.1 under the assumption that $I_{n+1}$ is not indiscernible over $B a$.

Proof. For each $k$ with $1 \leq k<n$ we will inductively define a "continuation" $I_{k}^{*}$ of $I_{k}$ in the following way:

Suppose we have picked $I_{j}^{*}$ for $j<k$, and define $I_{k}^{*}$ to be a sequence indexed by $\omega$ as in Proposition 3.3 for $I=I^{k}$ and $B=B \cup \bigcup_{i=1}^{n} I_{i} \cup \bigcup_{j=1}^{k-1} I_{j}^{*} \cup\{a\}$ so that given any subset $A \subseteq B \cup \bigcup_{i=1}^{n} I_{i} \cup \bigcup_{j=1}^{k-1} I_{j}^{*} \cup\{a\}$, the sequence $I_{k}^{\frown} I_{k}^{*}$ is indiscernible over $A$ whenever $I_{k}$ was indiscernible over $A$.

It follows from the construction and Corollary 3.4 that

- $\left\{I_{1} \frown I_{1}^{*}, \ldots, I_{n-1} \frown I_{n-1}^{*}, I_{n}, I_{n+1}\right\}$ is a set of mutually $B$-indiscernible sequences,

- $\left\{I_{1} \frown I_{1}^{*}, \ldots, I_{n-1} \frown I_{n-1}^{*}\right\}$ is mutually indiscernible over $I_{n+1} B a$, and

- $I_{n+1}$ is not indiscernible over $B a \bar{b}$ for some $\bar{b} \in \bigcup\left\{I_{1}, \ldots, I_{n-1}\right\}$. 
Since the sequences in $\left\{I_{1} \frown I_{1}^{*}, \ldots, I_{n-1} \frown I_{n-1}^{*}\right\}$ are mutually indiscernible over $I_{n+1} B a$, there is an automorphism fixing $I_{n+1} B a$ and sending $\bar{b}$ to some $\bar{b}^{\prime} \in$ $\bigcup\left\{I_{1}^{*}, \ldots, I_{n-1}^{*}\right\}$. Now we have

- $\left\{I_{1}, \ldots, I_{n-1}, I_{n}, I_{n+1}\right\}$ is a set of $B \bar{b}^{\prime}$-mutually indiscernible sequences,

- $\left\{I_{1}, \ldots, I_{n-1}\right\}$ is mutually indiscernible over $I_{n+1} B \bar{b}^{\prime} a$, and

- $I_{n+1}$ is not indiscernible over $B b^{\prime} a$,

which, replacing $B$ with $B \bar{b}^{\prime}$, is precisely the conditions we started with plus the conclusion of the claim. Since any $n$-subset of mutually $B b^{\prime} a$-indiscernible sequences of $\left\{I_{1}, \ldots, I_{n-1}, I_{n}, I_{n+1}\right\}$ would in particular be $B a$-indiscernible, the claim is proved.

Now the lemma follows almost immediately. Since $\left\{I_{2}, I_{3}, \ldots, I_{n}, I_{n+1}\right\}$ are mutually indiscernible over $I_{1} B$, there must, by induction hypothesis, be a subset of $n-1$ mutually $I_{1} B a$-indiscernible sequences. But such a set cannot contain $I_{n+1}$ since, by the hypothesis given in Claim 4.1.1, this sequence is not (by itself) indiscernible over $B a$. So $\left\{I_{2}, I_{3}, \ldots, I_{n}\right\}$ are mutually indiscernible over $I_{1} B a$. In exactly the same way we can prove that $\left\{I_{1}, I_{3}, \ldots, I_{n}\right\}$ are mutually indiscernible over $I_{2} B a$ which in particular implies that $I_{1}$ is indiscernible over $B \cup\left\{I_{2}, I_{3}, \ldots, I_{n}\right\} \cup\{a\}$. So $\left\{I_{1}, I_{2}, I_{3}, \ldots, I_{n}\right\}$ are mutually indiscernible over $B a$, as required.

Corollary 4.2 (Sub-additivity of dp-rank for dp-minimal types). Let $\operatorname{tp}\left(a_{i} / A\right)$ be $d p$-minimal for $1 \leq i \leq k$. Then the dp-rank of $\operatorname{tp}\left(a_{1} \ldots a_{k} / A\right)$ is at most $k$.

Proof. By induction on $k$. For $k=1$ there is nothing to do. Assume that the corollary holds for all sets $B$ and tuples of less than $k$ dp-minimal (over $B$ ) elements.

Now fix $A$ and $a_{1}, \ldots, a_{k}$ dp-minimal over $A$. Let $I_{1}, \ldots, I_{k}, I_{k+1}$ be mutually indiscernible over $A$. By the lemma above, without loss of generality, $I_{1}, \ldots, I_{k}$ are mutually indiscernible over $A a_{k}$; call it $B$.

Recall that extensions of dp-minimal types have rank at most 1 , so we may assume that $a_{1}, \ldots, a_{k-1}$ are dp-minimal over $B$. Hence by the induction hypothesis, dp-rank of the tuple $a_{1} \ldots a_{k-1}$ over $B$ is at most $k-1$. By definition, one of the sequences $I_{1}, \ldots, I_{k}$ is indiscernible over $B a_{1} \ldots a_{k-1}=A a_{1} \ldots a_{k}$, which is exactly what we needed.

Remark 4.3. Notice that in Claim 4.1.1 we did not assume that $I_{k}$ is indiscernible over $I_{n} \cup\{a\}$. That is, we know that $I_{k}$ (for $k<n$ ) is indiscernible over $B I_{\neq k}$ and over $B I_{\neq k, n} a$, but not necessarily $B I_{\neq k} a$. This (and the analogue issue in Lemma 4.6) is the reason we could not work using just Fact 3.1 and therefore decided to use the extensions described by Proposition 3.3 .

4.2. The finite case. The following proposition, from which the main result of this section will easily follow, is a generalization of Lemma 4.1.

Proposition 4.4. Let $a$ be an element such that $\operatorname{tp}(a / A)$ has dp-rank at most $k$, and let $\mathcal{I}:=\left\{I_{1}, \ldots, I_{m}\right\}$ be mutually $B$-indiscernible sequences with $m>k$. Then there is an $m-k$-subset of $\mathcal{I}$ of sequences which are mutually indiscernible over $B a$.

To prove Proposition 4.4 we rephrase the statement in a way that will allow us to do an easy induction. For this we will need the following definition. 
Definition 4.5. Let $\mathcal{I}:=\left\{I_{1}, \ldots, I_{m}\right\}$ be mutually $A$-indiscernible sequences, and let $a$ be any tuple. We will say that the pair $\mathcal{I}, a$ satisfies $S_{k, n}$ if the following conditions hold:

- $|\mathcal{I}| \geq k+n$.

- For any $B \supset A$ such that $\mathcal{I}:=\left\{I_{1}, \ldots, I_{m}\right\}$ are still mutually indiscernible over $B$, given any $n+k$ sequences in $\mathcal{I}$, at least $n$ of them remain mutually indiscernible over $B a$.

So, in particular, with this notation, a type $p(x)$ over $A$ has dp-rank less than or equal to $k$ if and only if for any realization $a$ of $p(x)$ and every set $\mathcal{I}$ of mutually indiscernible sequences where $|\mathcal{I}|>k$, we have that $\mathcal{I}$, $a$ satisfies $S_{k, 1}$.

With this notation we can state a generalization of Proposition 4.4 the proof of which will admit a clear induction argument. We will start by proving the following analogue of Claim 4.1.1.

Lemma 4.6. Let a be an element, and let $\mathcal{I}$ be a set of mutually A-indiscernible sequences. Let $\mathcal{J}$ be a subset of $\mathcal{I}$ and $I \in \mathcal{I}$ be such that $\mathcal{J}$ is mutually indiscernible over AIa and such that $I$ is not indiscernible over $A \mathcal{J} a$. Then we can extend $A$ to a set $B$ such that the following hold:

- I is mutually indiscernible over B.

- I is not indiscernible over Ba.

- $\mathcal{J}$ is mutually indiscernible over Ba.

Proof. We will assume that $\mathcal{J}$ is finite, which is the case we need for Theorem 4.8. However, the general case follows exactly in the same manner, using ordinal enumerations of the sequences in $\mathcal{J}$ and transfinite induction.

We can enumerate $\mathcal{J}:=\left\{J_{1}, \ldots, J_{n}\right\}$ and, as in the proof of Claim 4.1.1, define a "continuation" $J_{t}^{*}:=\left\langle a_{i}^{*}\right\rangle_{i \in \omega}$ for every sequence $J_{t} \in \mathcal{J}$ inductively (on $t$ ) having that $J_{t}^{\frown} J_{t}^{*}$ is indiscernible over any

$$
C \subseteq A \cup \bigcup \mathcal{I} \cup \bigcup_{j=1}^{t-1} J_{j}^{*} \cup\{a\}
$$

over which $J_{t}$ was already indiscernible.

Because $\mathcal{J}$ was mutually indiscernible over $A I a$ it follows from Corollary 3.4 that

- $\left\{J_{1}^{\frown} J_{1}^{*}, \ldots, J_{n}^{\frown} J_{n}^{*}\right\} \cup(\mathcal{I} \backslash \mathcal{J})$ is a set of $A$-mutually indiscernible sequences,

- $\left\{J_{1}^{\frown} J_{1}^{*}, \ldots, J_{n}^{\frown} J_{n}^{*},\right\}$ is indiscernible over $I A a$, and

- $I$ is not indiscernible over $A a \bar{b}$ for some $\bar{b} \in \bigcup\left\{J_{1}, \ldots, J_{n}\right\}$.

Since $\left\{J_{1}^{\frown} J_{1}^{*}, \ldots, J_{n}^{\frown} J_{n}^{*},\right\}$ is indiscernible over $I A a$ there is an automorphism fixing $I A a$ and sending $\bar{b}$ to some $\bar{b}^{\prime} \in \bigcup\left\{J_{1}^{*}, \ldots, J_{n}^{*}\right\}$. Now we have

- $\mathcal{I}$ is a set of $A \bar{b}^{\prime}$-mutually indiscernible sequences,

- $\left\{J_{1}, \ldots, J_{n}\right\}$ is indiscernible over $I A \bar{b}^{\prime} a$, and

- $I$ is not indiscernible over $A b^{\prime} a$.

Letting $B:=A b^{\prime}$ completes the claim.

Proposition 4.7. Let $a$ be an element, $n$ be any natural number, and let $\mathcal{I}:=$ $\left\{I_{1}, \ldots, I_{m}\right\}$ be mutually $A$-indiscernible sequences with $m \geq k+n$ such that $\mathcal{I}, a$ satisfies $S_{k, 1}$. Then $\mathcal{I}$, a satisfies $S_{k, n}$. 
Proof. Notice that we have already proved the result, assuming $k=1$. It is enough to show that $S_{k, n}$ implies $S_{k, n+1}$, and we will show this by induction on $n$ (for a fixed $k$ ).

So let $\mathcal{I}$ be a set of $m$ mutually $B$-indiscernible sequences, $a$ be an element such that $\mathcal{I}, a$ satisfies $S_{k, i}$ for all $1 \leq i \leq n$ (so, in particular, it satisfies $S_{k, n}$ and $S_{k, 1}$ ) and let $\mathcal{I}^{\prime}:=\left\{I_{1}, \ldots, I_{k+n+1}\right\}$ be a subset of $\mathcal{I}$. We will prove that $\mathcal{I}^{\prime}$ contains a subset of size $n+1$ of sequences which are mutually indiscernible over $B a$.

Let $I_{i}$ be any sequence in $\mathcal{I}^{\prime}$. Since $\mathcal{I}^{\prime} \backslash\left\{I_{i}\right\}$ is a set of $n+k$ mutually indiscernible sequences over $B I_{i}$, there is a subset $\mathcal{I}_{i}$ of size $n$ which are mutually indiscernible over $B I_{i} a$. If $I_{i}$ is indiscernible over $B \mathcal{I}_{i} a$, then we would have a set of size $n+1$ of mutually indiscernible sequences over $B a$ and the proposition would be satisfied. So we may assume towards a contradiction that for every $i$ the sequence $I_{i}$ is not indiscernible over $B \mathcal{I}_{i} a$.

Now, for each $i$ we apply Lemma 4.6. extending $B$ until we get $I_{i}$ not indiscernible over $B a$ for all $i$ and $\mathcal{I}^{\prime}$ are mutually indiscernible over $B$. This contradicts $S_{k, 1}$ of $\mathcal{I}$ (and $S_{k, n}$, too).

This completes the proof of Proposition 4.4.

Theorem 4.8. Let $a_{1}, a_{2}$ be tuples such that $\operatorname{rk}-\operatorname{dp}\left(\operatorname{tp}\left(a_{i} / A\right)\right) \leq k_{i}$ for $i \in\{1,2\}$. Then $\operatorname{rk}-\operatorname{dp}\left(\operatorname{tp}\left(a_{1}, a_{2} / A\right)\right) \leq k_{1}+k_{2}$.

Proof. Let $\mathcal{I}:=\left\{I_{1}, \ldots, I_{k_{1}+k_{2}+1}\right\}$ be mutually $A$-indiscernible sequences. By Proposition 4.4 applied to $a_{1}, \mathcal{I}$, there is a subset $\mathcal{I}_{1}$ of $\mathcal{I}$ of size $k_{2}+1$ of sequences which are mutually indiscernible over $A a_{1}$. By definition of dp-rank of $\operatorname{tp}\left(a_{2} / A a_{1}\right)$, we get that there is a sequence $I^{\prime} \in \mathcal{I}_{1}$ which is indiscernible over $A a_{1} a_{2}$. By definition of dp-rank, this completes the proof of the theorem.

We get the following corollary (compare with Corollary 4.12).

Corollary 4.9. Let $T$ be any theory.

If all the one variable types have finite $d p$-rank, then every type (with finitely many variables) in the theory has finite dp-rank.

If all the one variable types have $d p$-rank $\leq k$, then every type (with finitely many variables) $p(x)$ has $d p$-rank $\leq|x| \cdot k$. 2.8 .

The following corollary follows immediately from Theorem 4.8 and Proposition

Corollary 4.10. Let $T$ be any theory, and assume that all the one variable types have $d p$-rank $\leq k$. Then for every formula $\varphi(x, y)$ we have $\operatorname{alt}(\varphi(x, y)) \leq 2 k|x|+1$.

In particular, if $T$ is dp-minimal, then for every $\varphi(x, y)$ we have alt $(\varphi(x, y)) \leq$ $2|x|+1$.

4.3. The infinite case. The proof of sub-additivity for the infinite case is in fact much easier than in the finite case.

Theorem 4.11. Let $\mathcal{I}$ be a set of $\kappa$ mutually indiscernible sequences over $A$ and a be an element such that $S_{\kappa, 1}$ holds for $\mathcal{I}, a$. Then $S_{\kappa, \kappa}$ holds for $\mathcal{I}, a$. In particular, for any cardinal numbers $\kappa, \lambda$ and any tuples $a, b$ we have that

$$
\operatorname{rk}-\operatorname{dp}(a b / A) \leq \max (\kappa, \lambda)
$$

whenever $\operatorname{rk}-\operatorname{dp}(a / A) \leq \kappa$ and $\operatorname{rk}-\operatorname{dp}(b / A) \leq \lambda$. 
Proof. Let $\mathcal{I}, a$ be any pair satisfying $S_{\kappa, 1}$. We can partition $\mathcal{I}=\bigcup_{\alpha \in \kappa} \mathcal{I}^{\alpha}$ into a disjoint union of $\kappa$ many sets of $\kappa$ many sequences. By hypothesis we know that for any $\beta$ the set $\mathcal{I}^{\beta}$ is mutually indiscernible over $A \cup \bigcup_{\alpha \neq \beta} \mathcal{I}^{\alpha}$, so by assumption we have that some sequence $I^{\beta}$ in $\mathcal{I}^{\beta}$ is indiscernible over $A \cup \bigcup_{\alpha \neq \beta} \mathcal{I}^{\alpha} \cup\{a\}$. Doing this for any $\beta \in \kappa$ we get a set of sequences $\left\{I^{\alpha}\right\}_{\alpha \in \kappa}$ such that $I^{\beta}$ is indiscernible over $A \cup \bigcup_{\alpha \neq \beta} I_{\alpha} \cup\{a\}$, which by definition proves that $\mathcal{I}$, $a$ satisfies $S_{\kappa, \kappa}$. The rest of the proof follows exactly as in Theorem 4.8 .

Since this immediately implies that $\operatorname{rk}-\operatorname{dp}(a b / A) \leq \omega$ whenever $\operatorname{rk}-\operatorname{dp}(a / A) \leq \omega$ and $\operatorname{rk}-\operatorname{dp}(b / A) \leq \omega$, this theorem provides a very easy proof of the fact that strong dependence $(\operatorname{rk}-\operatorname{dp}(p(x)) \leq \omega$ for all $p(x)$ or, equivalently, no randomness pattern of depth $\omega$ ) only needs to be verified in one variable. Summarizing, we get the following (very) easy corollary, which was originally proved by Shelah in 12 . (Observation 1.6).

Corollary 4.12. A theory $T$ is strongly dependent if and only if all the one variable types in $T$ are strongly dependent.

Also, we get a dp-rank version of Shelah's theorem that $T$ is dependent if and only if the independence property cannot be witnessed by $\varphi(x, y)$ with $|x|=1$. Recall that a theory is dependent if and only if every type is dependent, that is, rk-dp $(p(x)) \leq|T|^{+}$for any type $p(x) \stackrel{2}{ }$ So the following, which follows immediately from Theorem 4.11, is a new (and simpler) proof of Shelah's Theorem II.4.11 in 9 .

Corollary 4.13. A theory $T$ is dependent (which is equivalent to every type being dependent) if and only if all the one variable types in $T$ are dependent.

\section{VC-DENSITY}

Recent results by Aschenbrenner, Dolich, Haskell, Macpherson and Starchenko 22 show that in many of the well-behaved dependent theories, the $V C$-density can be calculated and, in many of the cases that they considered, it is linear. In this section we define $V C$-density (or rather, a dual notion, which we call $V C^{*}$-density 3 ) and discuss some connections between it and dp-rank.

For the sake of clarity, we introduce some notation. Let $p(x)$ be a type and $B$ be a set; we denote the set of all complete types over $B$ which are consistent with $p(x)$ by $S^{p(x)}(B)$. If $\varphi(x, y)$ is a formula or $\Delta(x, y)$ is a set of formulas, we can speak of $\varphi(x, y)$-types, or $\Delta(x, y)$-types (over $B$ ) consistent with $p(x)$. These will be denoted by $S_{\varphi}^{p(x)}$ and $S_{\Delta}^{p(x)}$, respectively.

We also remind the reader of some basic notation for asymptotic behavior of functions. Let $f, g: \mathbb{N} \rightarrow \mathbb{R}_{+}$. We write that $f=O(g)$ if for some constant $r>0$ for any $n$ large enough we have $f(n) \leq r \cdot g(n)$.

Recall that if $f$ is a polynomial function, its order of magnitude is completely determined by its degree. That is, if $f, g$ are polynomials, then $f=O(g)$ if and only if $\operatorname{deg}(f) \leq \operatorname{deg}(g)$.

\footnotetext{
${ }^{2}$ We are defining a type to be dependent depending of the behavior of the "dual" formula. Reasons why this is the right notion can be found in Observation 2.7 in [7].

${ }^{3}$ Given a family $\Delta(x, y)$, the $V C_{\Delta}^{*}$-density will correspond to the $V C$-density of the family $\Delta$ after we invert the roles of $x$ and $y$.
} 
Definition 5.1. Let $\mathcal{C}$ be a large, saturated enough, model of $T$, let $\Delta(x, y)$ be a finite set of formulas in the language of $T$, and let $p(y)$ be a (partial) type over a set of parameters in $\mathcal{C}$. The $V C^{*}$-dimension of $p(y)$ with respect to $\Delta$ is greater than or equal to $n$ if there is a set $A$ of size $n$ such that, for any $A_{0} \subset A$, there is some $b \models p(y)$ and some $\delta(x, y) \in \Delta$ such that for any $a^{\prime} \in A$, we have

$$
\mathcal{C} \equiv \delta\left(a^{\prime}, b\right) \Leftrightarrow a^{\prime} \in A_{0} .
$$

Whenever this happens we will say that $\Delta$ shatters $A$ with realizations of $p(y)$.

We will say that the $V C^{*}$-dimension of $p(y)$ with respect to $\Delta$ is $n$ if the $V C^{*}$ dimension is greater than or equal to $n$ but not greater than or equal to $n+1$.

Notice that if $\Delta$ shatters a set $A$ with respect to $p(y) \in S_{k}(B)$, then every subset of $A$ is (externally) definable as

$$
\delta(\mathcal{C}, b) \cap A,
$$

where $b$ varies among realizations of $p(y)$ (and $\delta \in \Delta$ ). If $\Delta$ is a singleton (that is, if there is a single formula $\delta(x, y)$ in $\Delta)$, this is of course equivalent to saying that $p(y)$ is consistent with $2^{|A|}$ different $\Delta$-types over $A$. So, if instead of counting subsets we count types, we will get a notion that, although it is not exactly the same as $V C^{*}$-dimension when $\Delta$ is not a singleton, it is closely related to this notion (particularly asymptotically). Recall that by $S_{\Delta}^{p(y)}(A)$ we denote the set of all $\Delta$-types over $A$ consistent with $p(y)$. With this notation, we can look for the largest $n$ such that there is some set $A$ of size $n$ such that

$$
\left|S_{\Delta}^{p(y)}(A)\right| \geq 2^{|A|}
$$

We are slowly getting to the notion of $V C$-density with which we will work. It was proved (apparently independently by Sauer, Shelah, and Vapnik-Chervonenkis) that if the $V C^{*}$-dimension of $\Delta$ with respect to $p(y)$ is equal to $d$, then, for any $A$ of size greater than $d$, the number $\pi_{(\Delta, p)}(A)$ of subsets of $A$ externally definable with realizations of $p$ satisfies

$$
\pi_{\Delta, p}(A) \leq\left(\begin{array}{c}
|A| \\
0
\end{array}\right)+\cdots+\left(\begin{array}{c}
|A| \\
d
\end{array}\right)
$$

Now, $\left|S_{\Delta}^{p(y)}(A)\right|$ is bounded by $|\Delta|$ times $\pi_{\Delta, p}(A)$. This implies that when the $V C^{*}$-dimension of $\Delta$ with respect to $p$ is $d$, then each formula in $\Delta$ can define (using parameters in $p$ ) at most $O\left(|A|^{d}\right)$ externally definable sets of $A$. It follows that if we vary $A$ among increasing subsets of an infinite set, we get polynomial growth of the number of $\Delta$-types $\left|S_{\Delta}^{p(y)}(A)\right|$ over $A$, and a very natural question to ask is whether $d$ is the best bound on the degree of the polynomial. This prompts the following definition of $V C^{*}$-density of a type. We will define (adapting the notions in [2]) the $V C_{\Delta}^{*}$-density of a type $p(y)$ over a set $C$ to be

$$
\inf \left\{r \in \mathbb{R}^{\geq 0}|| S_{\Delta}^{p(y)}(A) \mid=O\left(|A|^{r}\right) \text { for all finite } A \subseteq C^{|y|}\right\} .
$$

What we formally mean by this is that there exists a function $f: \mathbb{N} \rightarrow \mathbb{R}_{+}$such that $f=O\left(n^{r}\right)$, and $\left|\mathrm{S}_{\Delta}^{p(y)}(A)\right| \leq f(|A|)$ for all $A \subseteq C$ finite.

If in the definition above $A$ is allowed to range over all finite sets (that is, $C=M$ for some $M \models T$ saturated enough), we omit "over $C$ " and simply say " $V C_{\Delta}^{*}$-density of $p$ ".

Using our present notation, Proposition 2.8 implies that if $\operatorname{rk}-\operatorname{dp}(p(y)) \geq k$, then there exists a formula $\varphi(x, y)$ and an indiscernible sequence $I$ in the sort of $y$ such 
that $\left|S_{\varphi}^{p(y)}\left(I^{\prime}\right)\right| \geq\left(\begin{array}{l}n \\ k\end{array}\right)$ for every $I^{\prime} \subseteq I$ of size $n \geq k$. This of course means that $V C_{\varphi}^{*}$-density of $p(y)$ is at least $k$. In order to make this connection between dp-rank and $V C^{*}$-density more precise, we state the following proposition.

Recall (Proposition 2.6) that $\operatorname{rk}-\operatorname{dp}(p) \geq k$ if and only if there is a randomness pattern $I_{\alpha}, \varphi_{\alpha}$ of depth $k$ for $p$. Below we will say that $\operatorname{rk}-\operatorname{dp}(p) \geq k$ is witnessed by formulas in $\Delta$ (where $\Delta$ is a set of formulas) if all $\varphi_{\alpha}$ are in $\Delta$.

Proposition 5.2. Let $p(y)$ be a type over $A$ and $\Delta$ be a set of formulas which is closed under boolean combinations. Then the following are equivalent.

(i) $\operatorname{rk}-\operatorname{dp}(p) \geq k$, witnessed by formulas in $\Delta$.

(ii) There is an $A$-indiscernible sequence $I$ and some formula $\varphi(x, y) \in \Delta$ such that $p(x)$ has $V C_{\varphi}^{*}$-density at least $k$ over $I$.

(iii) There is an $A$-indiscernible sequence $I$ and some formula $\varphi(x, y) \in \Delta$ such that $p(x)$ has $V C^{*}$-density larger than $k-1$ with respect to $\varphi(x, y)$ over $I$.

Proof. (i) $\Longrightarrow$ (ii) by Proposition 2.8, as explained above (note that the formula one gets in Proposition 2.8 is a boolean combination of $\Delta$-formulas, hence is itself in $\Delta$ ), and (ii) $\Longrightarrow$ (iii) is trivial.

(iii ) $\Longrightarrow($ i).

Let $f: \mathbb{N} \rightarrow \mathbb{N}$ be the following function: $f(n)=\left|S_{\varphi}^{p(y)}\left(I^{\prime}\right)\right|$ for some/every $I^{\prime} \subseteq I$ of size $n$. It follows from the assumption that $f$ is not $O\left(n^{k-1}\right)$. This means that for every $r>0$ there is $n$ such that $f(n)>r \cdot n^{k-1}$.

We may assume that the order type of $I$ is $\mathbb{R}$ (indeed, all we need is to keep $\left|S_{\varphi}^{p(y)}\left(I^{\prime}\right)\right|$ for all $I^{\prime} \subseteq I$ finite). We write $I=\left\langle a_{r}: r \in \mathbb{R}\right\rangle$.

In order to continue, we will need the following definition. Given some $c \models p$, an element $r \in \mathbb{R}$ will be defined to be a "switch point in $I$ for $c$ " if there is some $\epsilon \in \mathbb{R}$ such that either

$$
\varphi\left(c, a_{r-\delta}\right) \Leftrightarrow \neg \varphi\left(c, a_{r}\right)
$$

for all $\delta<\epsilon$ or

$$
\varphi\left(c, a_{r+\delta}\right) \Leftrightarrow \neg \varphi\left(c, a_{r}\right)
$$

for all $\delta<\epsilon$.

Claim 5.2.1. There is some $c=p$ for which there are at least $k$ switch points in $I$.

Proof of the claim. Assume this is not the case. Let $I^{\prime} \subseteq I$ be finite and denote $n=:\left|I^{\prime}\right|$. Let $q \in S_{\varphi}^{p(y)}\left(I^{\prime}\right), c=q$. Below we refer to a cut of $I^{\prime}$ (by which we mean either an element of $I^{\prime}$ or an interval between two adjacent elements in $I^{\prime}$ ) as a "switch cut for $c$ in $I^{\prime}$ " if the interval it induces in $I$ contains a switch point of $c$ in $I$. Note that there are $2 n+1$ cuts in $I^{\prime}$, hence at most $\left(\begin{array}{c}2 n+1 \\ k-1\end{array}\right)$ choices for possible sequences of switch cuts (by the assumption towards contradiction).

Notice that $q$ is completely determined by knowing (1) the switch cuts for $c$ in $I^{\prime},(2)$ what are the signs of $\varphi(c, y)$ for different segments between the switch cuts of $c$ in $I^{\prime}$, and (3) the sings of the cuts themselves. Since there are at most $\left(\begin{array}{c}2 n+1 \\ k-1\end{array}\right)$ choices for possible sequences of switch cuts, at most $2^{k}$ possible sequences of signs on the segments between the switch cuts, and at most $2^{k-1}$ possible signs for the switch cuts, we would get that $\left|\mathrm{S}_{\varphi}^{p(y)}\left(I^{\prime}\right)\right|=O\left(\left|I^{\prime}\right|^{k-1}\right)$ for all $I^{\prime}$, contrary to the assumption. 
Let $c \models p$ be such that there are at least $k$ switch points in $c$. We can then choose increasing indices $q_{0}, \ldots, q_{4 k-1}$ in $\mathbb{Q}$ such that for all $i<k$ the following hold:

- the closed $\mathbb{R}$-interval $\left[q_{4 i}, q_{4 i+1}\right]$ contains a switch point, and $\models \varphi\left(a_{q_{4 i}}, c\right)$ $\leftrightarrow \neg \varphi\left(a_{q_{4 i+1}}, c\right)$, and

- the open $\mathbb{R}$-interval $\left(q_{4 i+1}, q_{4(i+1)}\right)$ does not contain a switch point so that in particular $=\varphi\left(a_{q_{4 i+2}}, c\right) \leftrightarrow \varphi\left(a_{q_{4 i+3}}, c\right)$.

Now, let $J$ be the sequences of pairs of $I$

$$
J=\left\langle a_{q_{2 i}}, a_{q_{2 i+1}}: i<2 k\right\rangle,
$$

and let $\psi\left(x_{1}, x_{2} ; y\right)$ be the formula $\varphi\left(x_{1}, y\right) \leftrightarrow \varphi\left(x_{2}, y\right)$.

Clearly, $J$ is an $A$-indiscernible sequence, and since $\Delta$ is closed under boolean combinations, $\psi\left(x_{1}, x_{2}, y\right) \in \Delta$. Finally, by construction $\operatorname{alt}_{A}^{p(y)}(\psi)$ is at least $2 k$ witnessed by $J$, so by Proposition [2.8, we have $\operatorname{rk}-\operatorname{dp}(p) \geq k$, witnessed by $\psi \in \Delta$, as required.

Remark 5.3. Notice that we needed to define switch points, of which at first there may seem to be as many as the alternation rank. But there is the subtle issue that "isolated points" only count as one switch point, even though they contribute to two for the alternation rank. In fact, by changing the sequence and the formula, we manage to ensure that all those "switch points" happen on "isolated" points (now pairs), each of which then contributes two alternations, hence obtaining alternation rank $2 k$.

Proposition 5.2 explains why in the example of noninteger $V C$-density presented in 2] one has to work over sets that are not indiscernible, and why over indiscernible sequences $V C$-density becomes an integer; in this case, $V C$-density simply equals the appropriate dp-rank.

We now combine Propositions 2.6, 2.8, and 5.2, and summarize all the main characterizations of finite dp-rank that we have shown in this article.

Theorem 5.4. The following are equivalent for a type $p(y)$ over a set $A$ :

(i) $\operatorname{rk}-\mathrm{dp}(p) \geq k$.

(ii) There is a randomness pattern of depth $k$ for $p(x)$ over $A$.

(iii) There is a formula $\varphi(x, y)$ and an A-indiscernible sequence $I$ in the sort of $x$ such that the $V C_{\varphi}^{*}$-density of $p$ over $I$ is at least $k$.

(iv) There is a formula $\varphi(x, y)$ and an A-indiscernible sequence $I$ in the sort of $x$ such that the $V C_{\varphi}^{*}$-density of $p$ over $I$ is larger than $k-1$.

(v) There is a formula $\varphi(x, y)$ and an A-indiscernible sequence $I$ in the sort of $x$ such that for every $I^{\prime} \subseteq I$ of size $k$ there exists $c \models p$ satisfying $\varphi(x, c) \cap I=I^{\prime}$ (that is, every subset of I of size $k$ is externally $\varphi(x, y)$ definable by a realization of $p$ ).

(vi) There is a formula $\varphi(x, y)$ with $\operatorname{alt}_{A}^{p(y)}(\varphi) \geq 2 k$.

One may obtain a more precise (but also more technical) version of the theorem by restricting to a set of formulas $\Delta$ closed under boolean combinations, as in Proposition 5.2 
Remark 5.5. In the proof of (iii) $\Longrightarrow$ (i) in Proposition 5.2 we only needed that $\left|S_{\varphi}^{p(y)}\left(I^{\prime}\right)\right|$ is not $O\left(\left|I^{\prime}\right|^{k-1}\right)$, whereas the assumption gives more: it is not $O\left(\left|I^{\prime}\right|^{s}\right)$ for some $s>k-1$. Along this line, we note that by using clause (v) in Theorem 5.4 one can deduce that the following statements are also equivalent to $\operatorname{rk}-\mathrm{dp}(p) \geq k$. The statements are more technical, but the equivalences are stronger.

(1) There is $\varphi(x, y)$ and $I$ such that $\left|S_{\varphi}^{p(y)}\left(I^{\prime}\right)\right|=\Omega\left(\left|I^{\prime}\right|^{k}\right)$ for $I^{\prime}$ finite.

(2) There is $\varphi(x, y)$ and $I$ such that $\left|S_{\varphi}^{p(y)}\left(I^{\prime}\right)\right|=\omega\left(\left|I^{\prime}\right|^{k-1}\right)$ for $I^{\prime}$ finite.

(3) There is $\varphi(x, y)$ and $I$ such that $\left|S_{\varphi}^{p(y)}\left(I^{\prime}\right)\right|$ is not $O\left(\left|I^{\prime}\right|^{k-1}\right)$ for $I^{\prime}$ finite.

Where $f=\Omega(g)$ means $g=O(f)$, then $f=\omega(g)$ means that $f$ strictly dominates $g$ up to any multiplicative constant. That is, for every constant $r>0$ we have $f(n)>r \cdot g(n)$ for all $n$ large enough.

Note that in order to go from (3) to (1) one may need to change the formula and the indiscernible sequence (just as in the equivalence of (iv) and (v) in the theorem), and this is crucial. One may ask whether similar statements hold with the same formula and sequence. We have not given it much thought.

We have recently learned that Vincent Guingona and Cameron Hill have investigated $V C^{*}$-density (and other properties) over indiscernible sequences in much greater detail in 3 .

Notice that although Proposition 5.2 demonstrates a nice connection between dprank and $V C^{*}$-density, it is still quite unsatisfactory. One would hope to connect $V C^{*}$-density in general to dp-rank. For example, all known examples of dp-minimal theories seem to have $V C^{*}$-density 1 (most of what is known has been proved in [2]). Is this a coincidence or an example of a deep connection? Specifically, we ask:

Question 5.6. Does every dp-minimal theory have $V C^{*}$-density 1 ?

It is not very clear how to approach the general question. The proofs in 2 are very case-specific and difficult. Any statement which states a bound for the $V C^{*}$ density in terms of the dp-rank would need to involve achieving finite indiscernible sequences, and hence require nontrivial combinatorial arguments. Some partial results have been obtained by the authors in a subsequent work, but not much is known in general.

Thinking about the possible arguments, it came to our attention that things could be much more manageable if we could concentrate in single variables. By this we mean that both definitions of dp-rank and of $V C^{*}$-density could be made by looking at the behavior of the realizations of the type with respect to singletons (for precise statements, see the two questions that follow this discussion). This sort of result is not uncommon in model theory: A theory is dependent if arbitrarily large sets of elements (not tuples) cannot be shattered. If a dependent theory is unstable, then the strict order property can be witnessed with elements, etc. So it would not be too surprising if both $V C^{*}$-density and dp-rank could be defined by just looking at the singletons. The following question appeared in a first version of this paper.

Question 5.7. If $p(x)$ is a (partial) type over $A$ of $d p$-rank greater than $n$, can this be witnessed by indiscernible sequences of elements? This is, are there $I_{1}, \ldots, I_{n}$ mutually $A$-indiscernible sequences of singletons and some $c \models p(x)$ such that $I_{j}$ is not indiscernible over $A c$ for all $1 \leq j \leq n$ ? 
This was proved to be false: there are theories which are not dp-minimal but such that given any element and any two mutually indiscernible sequences of singletons (over the empty set), at least one of them is indiscernible over the element. However, since the theory is not dp-minimal, you can find a type over the empty set with dp-rank larger than 1 , thus providing a counterexample to the question even with $A=\emptyset$.

The question, however, turned out to be the wrong question. The following was proved by Kaplan and Simon in [5]:

Fact 5.8 ([5]). If $p(x)$ is a (partial) type over $A$ of $d p$-rank greater than $n$, there is an extension $q$ of $p$ over some $B \supset A$ such that $q$ has $d p$-rank greater than $n$, witnessed by indiscernible sequences of singletons.

The second question is concerned with the behavior of $V C^{*}$-density:

Question 5.9. Suppose that $p(y)$ is a type such that for all

$$
\Delta(x, y):=\left\{\delta_{1}(x, y), \delta_{2}(x, y), \ldots, \delta_{n}(x, y)\right\},
$$

where $x$ is a single variable, we have that the $V C^{*}$-density of $p(y)$ with respect to $\Delta$ is greater than $d$. Is $d$ the $V C^{*}$-density of $p(y)$ with respect to any $\Delta$ ?

Notice that a positive answer to Question 5.9 would imply that we could define the VC-density of a type by considering formulas $\Delta$ for which $\bar{x}$ is a singleton. If this were true, we would have more tools and evidence for establishing a tighter connection between dp-rank and $V C^{*}$-density.

\section{REFERENCES}

1. Hans Adler, Strong theories, burden, and weight, preprint (2007).

2. Matthias Aschenbrenner, Alfred Dolich, Dierdre Haskell, Dugald MacPherson, and Sergei Starchenko, Vapnik-chervonenkis density in some theories without the independence property, arXiv:1109.5438 (2011).

3. Vincent Guingona and Cameron Donnay Hill, Local dp-rank and vc-density over indiscernible sequences, arXiv:1108.2554 (2011).

4. Tapani Hyttinen, Remarks on structure theorems for $\omega_{1}$-saturated models, Notre Dame J. Formal Logic 36 (1995), no. 2, 269-278. MR1345748 (96g:03063)

5. Itay Kaplan and Pierre Simon, Unpublished notes (2011).

6. Itay Kaplan and Alexander Usvyatsov, Strict independence in dependent theories, preprint (2011).

7. Alf Onshuus and Alexander Usvyatsov, On dp-minimality, strong dependence, and weight, J. Symbolic Logic 76 (2011), no. 3, 737-758. MR2849244

8. ㄴ Thorn orthogonality and domination in unstable theories, Fund. Math. 214 (2011), no. 3, 241-268. MR2845725

9. S. Shelah, Classification theory and the number of nonisomorphic models, second ed., Studies in Logic and the Foundations of Mathematics, vol. 92, North-Holland Publishing Co., Amsterdam, 1990. MR.1083551 (91k:03085)

10. Saharon Shelah, Classification theory for elementary classes with the dependence property - a modest beginning, Sci. Math. Jpn. 59 (2004), no. 2, 265-316, Special issue on set theory and algebraic model theory. MR2062198(2005m:03063)

11. _ Dependent first order theories, continued, Israel J. Math. 173 (2009), 1-60. MR2570659

12. Strongly dependent theories, arXiv:0504197 (2009). 
13. Pierre Simon, On dp-minimal ordered structures, J. Symbolic Logic 76 (2011), no. 2, 448-460. MR2830411

14. Alexander Usvyatsov, Generically stable types in dependent theories, J. Symbolic Logic $\mathbf{7 4}$ (2009), no. 1, 216-250. MR2499428 (2010b:03037)

Mathematischen Instituts, Universität Münster, Einsteinstrasse 62, 48149 Münster, Germany

Departamento de Matemáticas, Universidad de los Andes, Cra 1 No. 18A-10, Edificio H, Bogotá, 111711, Colombia

URL: http://matematicas. uniandes.edu.co/aonshuus

Centro de Matemática e AplicaÇões Fundamentais, Universidade de Lisboa, Av. Prof. Gama Pinto, 2, 1649-003 Lisboa, Portugal

URL: http://ptmat.fc.ul.pt/〜alexus 